\title{
Independent and joint correlation of PNPLA3 I148M and TM6SF2 E167K variants with the risk of coronary heart disease in patients with non-alcoholic fatty liver disease
}

\author{
Jian-Ting Wu' ${ }^{1}$, Shou-Sheng Liu' ${ }^{2,3}$, Xiang-Jun Xie ${ }^{1}$, Qun Liu', Yong-Ning Xin ${ }^{3,4^{*}}$ (D) and Shi-Ying Xuan ${ }^{1 *}$
}

\begin{abstract}
Background: CHD is reported to be the primary cause of death in patients with NAFLD. Genetic susceptibility genes contribute to the developmental risk of NAFLD or CHD. Whether the genetic factors could affect the risk of CHD in NAFLD patients is not clear. The aim of this study was to investigate the association of PNPLA3 I148M and TM6SF2 E167K variants with the risk of CHD in NAFLD patients in Chinese Han population.

Patients and methods: PNPLA3 1148M and TM6SF2 E167K variants were genotyped in a cohort of 189 patients with NAFLD and CHD, as well as 242 patients with NAFLD and 242 healthy controls by gene sequencing. Additionally, serum lipids profiles were determined by standard clinical laboratory methods.

Results: The minor allele frequency of PNPLA3 I148M and TM6SF2 E167K were 0.39 and 0.06 in this cohort, respectively. The distributions of PNPLA3 $1148 \mathrm{M}$ genotypes and alleles were significant different in NAFLD group vs controls and in NAFLD+CHD group vs NAFLD group (all $P<0.05$ ). NAFLD patients who carry the CG + GG genotype suffered the relative lower risk of CHD than CC genotype carriers $(\mathrm{OR}=0.6,95 \% \mathrm{Cl}: 0.40-0.90, P=0.01)$. In addition, PNPLA3 I148M and TM6SF2 E167K possess the joint correlation with the decreased risk of CHD in NAFLD patients with the increased number of risk alleles. Besides, PNPLA3 I148M and TM6SF2 E167K variants associated with the decreased serum lipid levels in overall series.
\end{abstract}

Conclusions: There was a joint protective correlation of PNPLA3 I148M and TM6SF2 E167K variants with the developmental risk of CHD in NAFLD patients. PNPLA3 I148M and TM6SF2 E167K variants might correlated with the decreased risk of CHD in NAFLD patients by associated with the reduced serum lipid levels.

Keywords: PNPLA3 1148M, TM6SF2 E167K, Non-alcoholic fatty liver disease, Coronary heart disease

\section{Background}

Non-alcoholic fatty liver disease (NAFLD) is becoming the leading cause of chronic liver disease in the world [1]. In China, the prevalence of NAFLD was about $25 \%$ and has approximately doubled in the past two decades $[2,3]$. The typical characteristics of NAFLD patients

\footnotetext{
*Correspondence: xinyongning@163.com; xuansydxy@163.com

${ }^{3}$ Digestive Disease Key Laboratory of Qingdao, Qingdao 266071, China

'Department of Gastroenterology, Qingdao Municipal Hospital, Qingdao University, Qingdao 266011, China

Full list of author information is available at the end of the article
}

include obesity, insulin resistance, higher serum lipid profiles, and abundant of NAFLD patients possess the diabetes and hyperlipemia [4]. In Epidemiological studies, NAFLD had been proven as an increased risk of atherosclerosis and cardiovascular disease. Some experts even proposed that NAFLD is the causal risk of coronary heart disease (CHD), despite both the risk factors of them were different $[5,6]$. Therefore, exploring the CHD risk in NAFLD patients and identifying the risk factors are necessary to manage and prevent the development of CHD in NAFLD patients. 
Genetic factor is the significant risk factors for the development of NAFLD, which had been studies for many years [7]. In 2008, Romeo et al. firstly reported the key role of PNPLA3 I148M in NAFLD patients by genome-wide association study (GWAS), which described that PNPLA3 I148M variant conferred to the higher hepatic fat content and severe hepatic inflammation [8]. In 2014, Kozlitina et al. founded that TM6SF2 E167K was another significant polymorphism site for the risk of NAFLD by GWAS [9]. Subsequently, many studies had investigated the roles of PNPLA3 I148M and TM6SF2 E167K in different countries and ethnic, and the results also proven that PNPLA3 I148M and TM6SF2 E167K variants were the risk factors for the development of NAFLD [10-13]. In addition, PNPLA3 I148M and TM6SF2 E167K variants were found to have additive effect on increasing the NAFLD risk in Chinese Han population [14, 15]. Recently, some studies revealed the independent effect of PNPLA3 I148M and TM6SF2 E167K on the risk of cardiovascular disease. The positive association of PNPLA3 I148M with the premature CHD in T2DM patients was observed, and Ruschenbaum et al. found that PNPLA3 I148M is associated with a relatively benign $\mathrm{CHD}$ risk in German. Pirola conducted a metaanalysis to investigate the role of TM6SF2 E167K in patients with NAFLD and CHD, and they found that TM6SF2 E167K may possess the dual and opposite role in protecting against CHD and increase the risk of NAFLD [16-18].

In consideration of the tightly association of NAFLD and CHD in patients, and PNPLA3 I148M and TM6SF2 E167K variants may possess the joint effect to conferred to the higher risk of NAFLD, it is meaningful to investigate the correlation of PNPLA3 I148M and TM6SF2 E167K variants with the development risk of CHD in NAFLD patients. The aim of this study was to explore the relationship of PNPLA3 I148M and TM6SF2 E167K variants with the risk of CHD in NAFLD patients in Chinese Han population, and explore the effects of PNPLA3 I148M and TM6SF2 E167K variants on the serum lipid profiles in overall series.

\section{Patients and methods}

\section{Study subjects}

This study was conducted according to the principles of Helsinki declaration and its appendices [19], and were approved by the Ethical Committee of Qingdao Municipal Hospital (Qingdao, China). All the subjects had signed the informed consent before participating in this study. All the subjects were recruited from June 2018 to March 2019, which include 266 healthy controls, 242 NAFLD patients diagnosed by B-type ultrasonography, and 189 patients with NAFLD and CHD (NAFLD+CHD) who undergone elective coronary angiography. All the subjects were Chinese Han population who had the normal diet and moderate exercise. The age and sex of all the recruited subjects were matched. Subjects as below were excluded: 1) possessing a history of heart failure; 2) long-term consumption of alcohol (males > $210 \mathrm{~g} / \mathrm{w}$, females $>140 \mathrm{~g} / \mathrm{w}$ ) or cigarettes; 3) accompanied with the viral hepatitis, autoimmune hepatitis, drug-induced hepatitis, or various liver cirrhosis; 4) suffering from tumors or surgery in nearly 2 years; 5 ) undergone any treatment for NAFLD or CHD.

\section{Biochemical analyses}

Blood samples of each subject were taken after a 12-h overnight fasting and placed into an ethylene diamine tetraacetic acid (EDTA)-containing tube. The serum clinical parameters such as triglyceride (TG), total cholesterol (TC), low-density lipoprotein (LDL), high-density lipoprotein (HDL), Alanine aminotransferase (ALT), aspartate aminotransferase (AST), gamma-glutamyltranspeptidase (GGT), alkaline phosphatase (ALP), fasting plasma glucose (FPG), total bilirubin (TBIL) were measured by standard clinical laboratory techniques. The basic information such as name, gender, age, body height and weight were acquired by a standard study questionnaire. Body mass index (BMI) was calculated as the mass $(\mathrm{kg}) /$ height $(\mathrm{m})^{2}$.

\section{Genomic DNA extraction and genotyping}

Genomic DNA was extracted from blood samples with the DNA extraction Kit (TIANGEN, Beijing, China) and stored at $-20^{\circ} \mathrm{C}$ until use. Polymerase chain reaction (PCR) was used for the genotyping of PNPLA3 I148M and TM6SF2 E167K with the primers: 5'-AACTTC TCTCTCCTTTGCTTTCACA-3' and 5'- GGAGGG ATAAGGCCACTGTAGA-3' for PNPLA3 I148M; 5'TGTCTCAGAACAAACAAACAAACAGA-3' and 5'-

Table 1 Clinical and biochemical characteristics of each group

\begin{tabular}{llll}
\hline Characteristic & $\begin{array}{l}\text { NAFLD }+ \text { CHD } \\
(n=189)\end{array}$ & $\begin{array}{l}\text { NAFLD } \\
(n=242)\end{array}$ & $\begin{array}{l}\text { Control } \\
(n=266)\end{array}$ \\
\hline Age, y & $58.10 \pm 6.95$ & $57.7 \pm 8.53$ & $58.90 \pm 5.53$ \\
$\mathrm{BMI}, \mathrm{kg} / \mathrm{m}^{2}$ & $25.69 \pm 3.2^{*} @$ & $26.76 \pm 2.82^{*}$ & $23.65 \pm 3.50$ \\
$\mathrm{FPG}, \mathrm{mmol} / \mathrm{L}$ & $5.81 \pm 1.81^{* @}$ & $5.00 \pm 1.35$ & $4.85 \pm 1.55$ \\
$\mathrm{ALT}, \mathrm{U} / \mathrm{L}$ & $30.23 \pm 2.80^{*}$ & $34.56 \pm 26.27^{*}$ & $22.47 \pm 21.14$ \\
$\mathrm{AST}, \mathrm{U} / \mathrm{L}$ & $45.73 \pm 70.71^{*} @$ & $28.45 \pm 21.49^{*}$ & $22.52 \pm 11.84$ \\
$\mathrm{TC}, \mathrm{mmol} / \mathrm{L}$ & $5.22 \pm 1.17$ & $5.48 \pm 0.83^{*}$ & $5.10 \pm 1.17$ \\
$\mathrm{TG}, \mathrm{mmol} / \mathrm{L}$ & $2.00 \pm 1.07^{*}$ & $1.92 \pm 1.31^{*}$ & $1.52 \pm 1.10$ \\
$\mathrm{LDL}, \mathrm{mmol} / \mathrm{L}$ & $3.09 \pm 1.06$ & $3.26 \pm 0.59$ & $3.15 \pm 0.73$ \\
$\mathrm{HDL}, \mathrm{mmol} / \mathrm{L}$ & $1.03 \pm 0.28^{*} @$ & $1.23 \pm 0.21$ & $1.29 \pm 0.38$ \\
\hline
\end{tabular}

Abbreviations: NAFLD non-alcoholic fatty liver disease, $C H D$ coronary heart disease, BMI body mass index, FPG fasting plasma glucose, $T C$ total cholesterol, $T G$ triglyceride, $L D L$ low-density lipoprotein cholesterol, HDL high-density lipoprotein cholesterol, AST aspartate aminotransferase, ALT

alanine aminotransferase

* Compared with the control group, $P<0.05$; ${ }^{\circledR}$ compared with NAFLD group, $P<0.05$

${ }^{\mathrm{a}}$ Data are presented as mean $\pm \mathrm{SD}$ 
Table 2 Minor allele frequency in the present study and other populations

\begin{tabular}{llllll}
\hline SNP & Present study & AFR & AMR & EAS & EUR \\
\hline PNPLA3 1148M & 0.39 & 0.12 & 0.48 & 0.35 & 0.23 \\
TM6SF2 E167K & 0.06 & 0.02 & 0.06 & 0.09 & 0.07 \\
\hline
\end{tabular}

Abbreviation: AFR African, AMR American, EAS East Asian, EUR European

GTAGGgGATGGTGAGgAAGAAG - 3' for TM6SF2 E167K. The PCR amplification reaction was conducted as the following procedure: $95^{\circ} \mathrm{C}$ for $5 \mathrm{~min}, 40$ cycles before denaturation at $94{ }^{\circ} \mathrm{C}$ for $30 \mathrm{~s}$, annealing at $58^{\circ} \mathrm{C}$ for $30 \mathrm{~s}$ and elongation $30 \mathrm{~s}$ at $72^{\circ} \mathrm{C}$. The genotypes of PNPLA3 I148M and TM6SF2 E167K variants were detected by ABI3730XL (Foster City, CA, USA) and then calculated by Gene Mapper 4.1 software.

\section{Statistical analysis}

SPSS 24.0 statistical software was used for statistical analysis. The Hardy-Weinberg equilibrium was measured using $X^{2}$ test. The difference of Genotype and allele distributions in each group was assessed by $X^{2}$ test and Fisher's exact test where appropriate. Associations between the PNPLA3 I148M, TM6SF2 E167K and NAFLD+CHD patients were assessed in logistic regression models. SNPs were analyzed using an additive model (by coding the genotype 0,1 and 2 for CC, CG and GG respectively) for PNPLA3 gene and a dominant model (by coding the genotype 0 and 1 for $\mathrm{CC}$ and $\mathrm{CT}+$ TT respectively) for TM6SF2 gene. The lipids characteristics among each group were shown as mean \pm SD and the differences was examined using student's $t$ test. The $P$ values less than 0.05 were considered to be statistically significant.

\section{Results}

\section{Clinical characteristics of the study subjects}

Table 1 shows the characteristics of healthy controls, NAFLD patients and NAFLD+CHD patients. As the results shown, the age and sex of subjects in each group were all matched. The BMI values and serum AST levels in NAFLD+CHD patients were higher than in NAFLD patients and health controls, and the BMI values and serum AST levels in NAFLD group was also higher than in health controls (all $P<0.05$ ). There were significant difference of serum FPG and HDL levels in NAFLD + CHD patients compared to NAFLD patients or health controls (all $P<0.05$ ), but no obviously difference was observed between NAFLD patients and health controls $(P>0.05)$. In addition, serum ALT and TG levels in NAFLD $+C H D$ patients and NAFLD patients were significantly higher than health controls (all $P<0.05$ ).

\section{Genotype and allele distributions of PNPLA3 I148M and TM6SF2 E167K}

The genotype distributions of the two SNPs (PNPLA3 I148M and TM6SF2 E167K) were conforming to the Hardy-Weinberg equilibrium in each group $\left(P_{\text {NAFLD }+}\right.$ $\mathrm{CHD}=0.92,0.98 ; P_{\mathrm{NAFLD}}=0.99,0.63 ; P_{\text {control }}=0.87,0.60$, respectively). The minor allele frequency (MAF) of PNPLA3 I148M and TM6SF2 E167K genotyped in our study were comparable to the overall frequency in east Asians, all the allele frequency information were collected from 1000 Genomes Project [20] (Table 2).

\section{Independent correlation of PNPLA3 I148M with the risk of CHD in NAFLD patients}

Table 3 demonstrated the genotype and allele distributions of PNPLA3 I148M in NAFLD+CHD patients, NAFLD patients and healthy controls. As the results shown, the genotype and allele distributions of PNPLA3 I148M were significant different in NAFLD+CHD vs NAFLD groups and in NAFLD vs control groups. In the Table 4, the results showed that the genotype CG + GG could increase the risk of NAFLD significantly compared to controls (OR $=1.53,95 \% \mathrm{CI}$ : $1.06-2.22, P=0.02)$. However, the CG + GG genotype could decrease the risk of CHD in NAFLD patients (OR $=0.60,95 \% \mathrm{CI}: 0.40-0.90$, $P=0.01$ ), which suggested the protective role of PNPLA3 I148M for the risk of CHD in NAFLD patients.

Table 3 Genotype and allele frequency of PNPLA3 1148M in each group

\begin{tabular}{|c|c|c|c|c|c|c|c|c|c|}
\hline \multirow[t]{2}{*}{$\overline{S N P}$} & \multirow{2}{*}{$\begin{array}{l}\text { NAFLD+CHD } \\
(n=189)\end{array}$} & \multirow{2}{*}{$\begin{array}{l}\text { NAFLD } \\
(n=242)\end{array}$} & \multirow{2}{*}{$\begin{array}{l}\text { Control } \\
(n=266)\end{array}$} & \multicolumn{2}{|c|}{ NAFLD+CHD vs NAFLD } & \multicolumn{2}{|c|}{ NAFLD+CHD vs Control } & \multicolumn{2}{|c|}{ NAFLD vs Control } \\
\hline & & & & $\overline{x^{2}}$ & $P$ value & $\overline{x^{2}}$ & $P$ value & $\overline{x^{2}}$ & $P$ value \\
\hline \multicolumn{10}{|c|}{ PNPLA3 I148M } \\
\hline Genotype & & & & 9.30 & 0.01 & 0.21 & 0.90 & 8.36 & 0.02 \\
\hline $\mathrm{CC}$ & 79 & 73 & 106 & & & & & & \\
\hline CG & 89 & 121 & 128 & & & & & & \\
\hline GG & 21 & 48 & 32 & & & & & & \\
\hline Allele & & & & 9.13 & 0.00 & 0.09 & 0.77 & 4.69 & 0.03 \\
\hline C & 247 (65.34) & $267(55.17)$ & $340(63.90)$ & & & & & & \\
\hline G & $131(34.66)$ & $217(44.83)$ & $192(36.09)$ & & & & & & \\
\hline
\end{tabular}


Table 4 Association between PNPLA3 I148M and CHD outcome in NAFLD patients

\begin{tabular}{|c|c|c|c|c|c|c|}
\hline \multirow{2}{*}{$\begin{array}{l}\text { PNPLA3 } \\
\text { I148M }\end{array}$} & \multicolumn{2}{|c|}{ NAFLD+CHD vs NAFLD } & \multicolumn{2}{|c|}{ NAFLD+CHD vs control } & \multicolumn{2}{|l|}{ NAFLD vs control } \\
\hline & OR $(95 \% \mathrm{Cl})$ & $P$ value & OR $(95 \% \mathrm{Cl})$ & $P$ value & OR $(95 \% \mathrm{Cl})$ & $P$ value \\
\hline CC & 1 & & 1 & & 1 & \\
\hline$C G+G G$ & $0.60(0.40-0.90)$ & 0.01 & $0.92(0.63-1.35)$ & 0.68 & $1.53(1.06-2.22)$ & 0.02 \\
\hline
\end{tabular}

Additive correlation of PNPLA3 I148M and TM6SF2 E167K with the risk of CHD in NAFLD patients

We further investigated the join correlation of PNPLA3 I148M and TM6SF2 E167K with the outcome of CHD in NAFLD patients. Figure 1 showed the proportion of the patients with NAFLD and CHD harboring different number of risk alleles. In our study, we didn't observe any individual with four SNPs. The proportion of patients with NFALD+CHD decreased significantly along with the increase of the number of risk alleles $\left(\chi^{2}=270\right.$, $P<0.01)$. As the Table 5 shown, the increase of the number of risk alleles showed a strong protective correlation with the CHD risk in NAFLD patients.

\section{Metabolic traits of PNPLA3 I148M and TM6SF2 E167K}

The difference of serum lipid levels between subjects with PNPLA3 CC genotype and CG + GG genotype was shown in the Fig. 2. We found that subjects with $\mathrm{CC}$ genotype possess the higher levels of TG and LDL and the lower level of HDL (all $P<0.05$ ). No significant difference of $\mathrm{TC}$ level was observed between the two

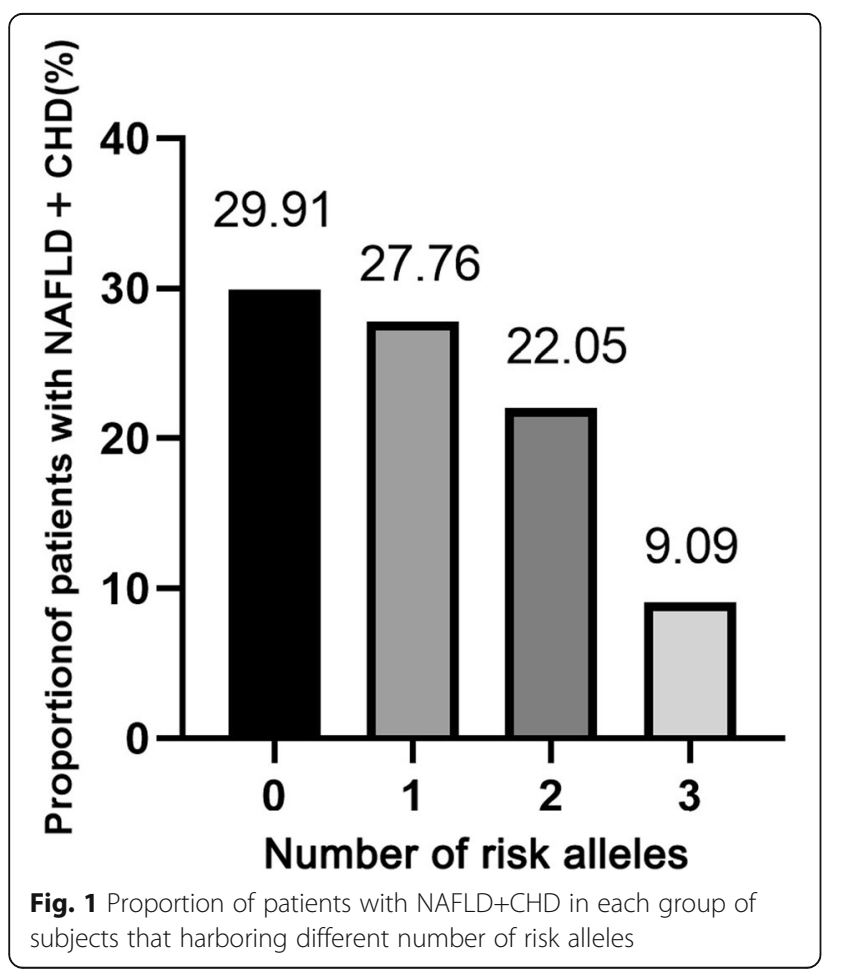

groups $(P>0.05)$. We further analyzed the serum levels of TC, TG, HDL, and LDL in subjects with different number of risk alleles. As shown in the Fig. 3, the serum levels of TG and LDL were decreased significantly with the increase of number of risk alleles. In addition, the serum level of HDL was increased significantly with the increase of number of risk alleles. No obvious difference was observed of the serum TC level in each group of subjects that harboring different number of risk alleles.

\section{Discussion}

Previous epidemiological studies reported that NAFLD was associated with an increased risk of $\mathrm{CHD}$, thereby put forward the hypothesis that NAFLD might be a causal risk factor for CHD [5, 6], In addition, CHD is the primary cause of death in patients with NAFLD [21]. To identify the CHD risk in NAFLD patients might be helpful in improving the management of NAFLD and CHD. Previous study had proven that PNPLA3 I148M and TM6SF2 E167K variants possess the additive effect on the risk of NAFLD [15]. In this study, we investigated the association of PNPLA3 I148M and TM6SF2 E167K variants with the risk of CHD in NAFLD patients for the first time. We counted the Minor allele frequency of PNPLA3 I148M and TM6SF2 E167K variant in this study, and the results were accorded with the mutation rate in East Asian. According to the results, PNPLA3 I148M variant was significant associated with the decreased risk of CHD in NAFLD patients. The possible reason is that PNPLA3 I148M could decrease the serum lipids levels in NAFLD, thereby lead to the decreased risk of CHD.

Several studies had shown that PNPLA3 rs738409 G allele might be associated with a relatively benign cardiovascular risk due to the tightly association with lower serum LDL level, which is the high risk factor for

Table 5 Risk for CHD outcome in NAFLD patients with different number of SNPS

\begin{tabular}{lll}
\hline Number of risk alleles & OR $(95 \% \mathrm{Cl})$ & $P$ value \\
\hline 0 & 1 & \\
1 & $0.632(0.405-0.987)$ & 0.04 \\
2 & $0.418(0.235-0.743)$ & $<0.01$ \\
3 & $0.119(0.014-1.000)$ & 0.05 \\
\hline
\end{tabular}



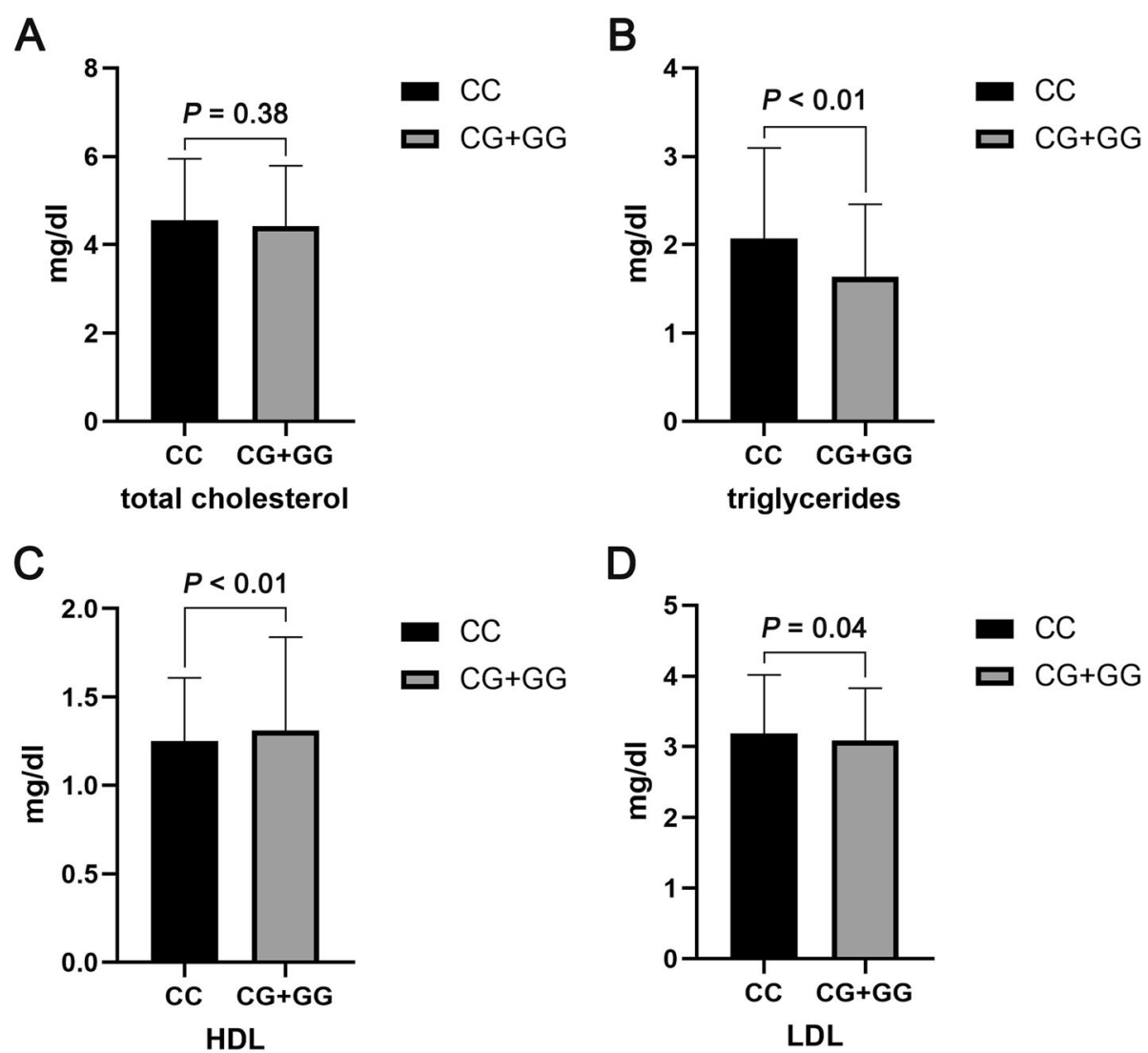

D

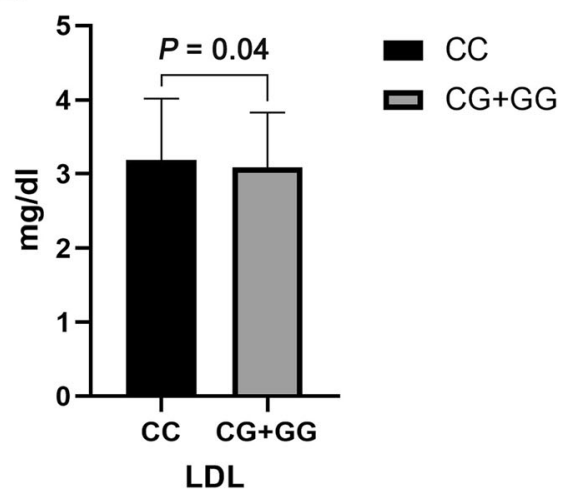

Fig. 2 Differences of serum levels of (a) TC, (b) TG, (c) HDL, and (d) LDL between subjects with PNPLA3 CC genotype and CG + GG genotype. Data are presented as mean values and standard deviations (Mean \pm SD)

cardiovascular $[9,22]$. In this study, we analyzed the correlation of PNPLA3 I148M variant with the serum lipid levels in overall series. We found that the serum levels of TG and LDL were significantly lower and the serum HDL levels were higher in subjects with CC genotype compared to CG + GG genotype carriers. These results suggested that PNPLA3 CG + GG genotype might tightly associate with the decreased serum lipid levels in overall series, which in accordance with the previous report [22]. In addition, some reports proposed that TM6SF2 E167K could decrease the plasma levels of LDL and TC, therefore, we also investigate the joint correlation of PNPLA3 I148M and TM6SF2 E167K variant with the risk of CHD in NAFLD patients and serum lipid levels in overall series. We found the risk of CHD in NAFLD patients was decreased along with the increase of risk alleles. In the overall series, the proportion of patients with NAFLD+CHD was decreased along with the increase of risk alleles of PNPLA3 I148M and TM6SF2 E167K. The serum TG and LDL levels were reduced, and serum HDL levels were decreased along with the increase of risk alleles. These results suggested that PNPLA3 I148M and TM6SF2 E167K possess the joint correlation with the decreased risk of CHD in NAFLD patients, and were associated with the decreased serum lipid levels in overall series.

There were some limitations in this study. Firstly, ultrosonography was performed to diagnosis of NAFLD other than liver biopsy. Secondly, the size of subjects in this study may not sufficiently large to explore the association between PNPLA3 I148M and TM6SF2 E167K with the risk of CHD in NAFLD patients. Thirdly, previous studies had showed that PNPLA3 I148M and TM6SF2 E167K variants were strongly associated with the decreasing kidney function, which impacts on the development of CHD [23-25], this factor was not taken into consideration.

\section{Conclusion}

In summary, we investigated the correlation of PNPLA3 I148M and TM6SF2 E167K variants with the risk of CHD in NAFLD patients. The PNPLA3 I148M was associated with the decreased risk of CHD in NAFLD patients, and was associated with the decreased the serum lipid levels in overall series. PNPLA3 I148M and TM6SF2 E167K variants were tightly correlated with the 


\section{A total cholesterol}


B
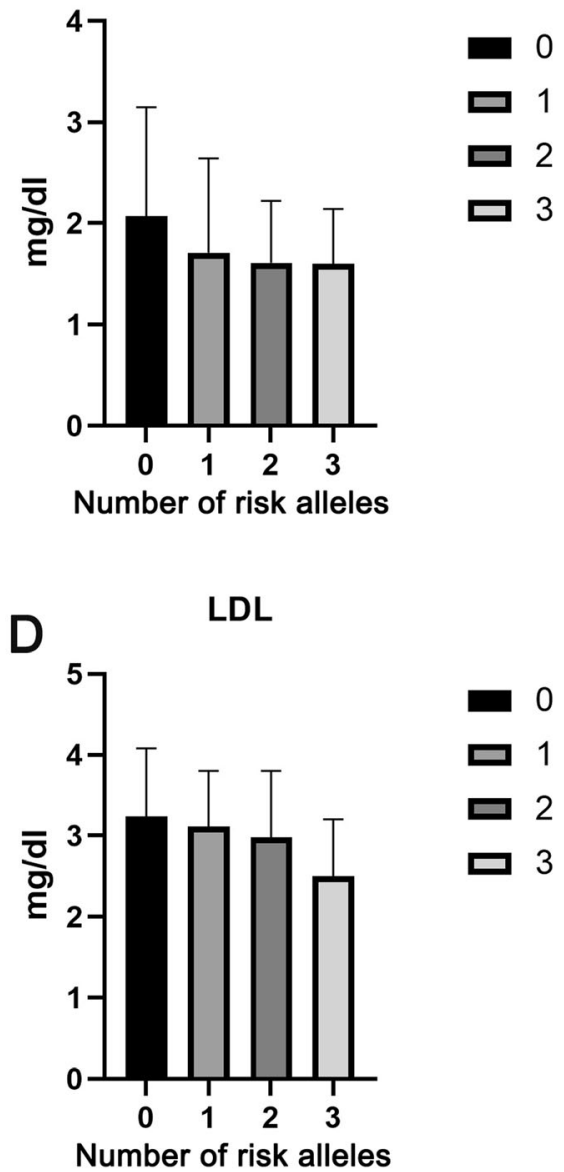

Fig. 3 Differences of serum levels of (a) TC, (b) TG, (c) HDL, and (d) LDL between subjects with different number of risk alleles. Data are presented as mean values and standard deviations (Mean \pm SD)

decreased risk of CHD in NAFLD patients and the decreased serum lipid levels in overall series along with the increased numbers of risk alleles. These results suggested that genetic factor such as PNPLA3 I148M and TM6SF2 E167K are very important in the developmental risk of CHD in NAFLD patients, which could be regarded as the potential diagnostic biomarkers for the risk assessment of CHD in NAFLD patients.

\section{Abbreviations}

ALP: Alkaline phosphatase; ALT: Alanine aminotransferase; AST: Aspartate aminotransferase; BMI: Body mass index; CHD: Coronary heart disease; Cl: Confidence intervals; EDTA: Ethylene diamine tetraacetic acid; FPG: Fasting plasma glucose; GGT: Gamma-glutamyltranspeptidase; GWAS: Genome-wide association studies; HDL: High density lipoprotein; LDL: Low density lipoprotein; NAFLD: Non-alcoholic fatty liver disease; ORs: Odds ratios; PCR: Polymerase chain reaction; SNP: Single nucleotide polymorphism; TBIL: Total bilirubin; TC: Total cholesterol; TG: Triglycerides; TRIB1: Tribbles-1

\section{Acknowledgements}

We thank the colleagues of Department of Infectious Disease for the help in this study.
Authors' contributions

Study concept and design: XYN and XSY. Subjects collection: WJT, LSS, XXJ, and LQ. Acquisition and analysis of data: WJT and LSS. The drafting and writing of the manuscript: WJT and LSS. The revision of the manuscript: XYN and XSY. All authors approved the final manuscript.

\section{Funding}

This study was supported by Grants of National Natural Science Foundation of China (31770837) and the Qingdao People's Livelihood Science and technology plan (18-6-1-68-nsh).

Availability of data and materials

The data and materials are available from the corresponding author for the reasonable request.

\section{Ethics approval and consent to participate}

The study protocol was approved by the Ethics Committee of Qingdao Municipal Hospital before participation (Approval NO.2017-20). All the subjects have signed written informed consent.

Consent for publication Not applicable.

Competing interests

The authors declare that they have no competing interests. 


\section{Author details}

'Department of Gastroenterology, Qingdao Municipal Hospital, Qingdao University, Qingdao 266011, China. ${ }^{2}$ Central Laboratories, Qingdao Municipal Hospital, Qingdao University, Qingdao 266071, China. ${ }^{3}$ Digestive Disease Key Laboratory of Qingdao, Qingdao 266071, China. ${ }^{4}$ Department of Infectious Disease, Qingdao Municipal Hospital, Qingdao University, Qingdao 266011, China.

Received: 31 October 2019 Accepted: 18 February 2020

Published online: 24 February 2020

\section{References}

1. Guerrero R, Vega GL, Grundy SM, Browning JD. Ethnic differences in hepatic steatosis: an insulin resistance paradox? Hepatology. 2009;49:791-801.

2. Fan JG. Epidemiology of alcoholic and nonalcoholic fatty liver disease in China. J Gastroenterol Hepatol. 2013;28(Suppl 1):11-7.

3. Fan JG, Kim SU, Wong WW. New trends on obesity and NAFLD in Asia. J Hepatol. 2017:67:862-73.

4. Romero-Gomez M, Zelber-Sagi S, Trenell M. Treatment of NAFLD with diet, physical activity and exercise. J Hepatol. 2017:67:829-46.

5. Lonardo A, Sookoian S, Pirola CJ, Targher G. Non-alcoholic fatty liver disease and risk of cardiovascular disease. Metabolism. 2016;65:1136-50

6. Targher G, Day CP, Bonora E. Risk of cardiovascular disease in patients with nonalcoholic fatty liver disease. N Engl J Med. 2010;363:1341-50.

7. Arab JP, Arrese M, Trauner M. Recent insights into the pathogenesis of nonalcoholic fatty liver disease. Annu Rev Pathol. 2018;13:321-50.

8. Romeo S, Kozlitina J, Xing C, Pertsemlidis A, Cox D, Pennacchio LA, Boerwinkle $\mathrm{E}$, Cohen JC, Hobbs HH. Genetic variation in PNPLA3 confers susceptibility to nonalcoholic fatty liver disease. Nat Genet. 2008:40:1461-5.

9. Kozlitina J, Smagris E, Stender S, Nordestgaard BG, Zhou HH, TybjaergHansen A, Vogt TF, Hobbs HH, Cohen JC. Exome-wide association study identifies a TM6SF2 variant that confers susceptibility to nonalcoholic fatty liver disease. Nat Genet. 2014;46:352-6.

10. Liu YL, Reeves HL, Burt AD, Tiniakos D, McPherson S, Leathart JB, Allison ME, Alexander GJ, Piguet AC, Anty R, et al. TM6SF2 rs58542926 influences hepatic fibrosis progression in patients with non-alcoholic fatty liver disease. Nat Commun. 2014:5:4309.

11. Zhou Y, Llaurado G, Oresic M, Hyotylainen T, Orho-Melander M, Yki-Jarvinen $\mathrm{H}$. Circulating triacylglycerol signatures and insulin sensitivity in NAFLD associated with the E167K variant in TM6SF2. J Hepatol. 2015;62:657-63.

12. Dai G, Liu P. Li X, Zhou X, He S. Association between PNPLA3 rs738409 polymorphism and nonalcoholic fatty liver disease (NAFLD) susceptibility and severity: a meta-analysis. Medicine (Baltimore). 2019:98:e14324.

13. Zhang L, You W, Zhang H, Peng R, Zhu Q, Yao A, Li X, Zhou Y, Wang X, Pu L, Wu J. PNPLA3 polymorphisms (rs738409) and non-alcoholic fatty liver disease risk and related phenotypes: a meta-analysis. J Gastroentero Hepatol. 2015;30:821-9.

14. Wang X, Liu Z, Wang K, Wang Z, Sun X, Zhong L, Deng G, Song G, Sun B, Peng $Z$, Liu W. Additive effects of the risk alleles of PNPLA3 and TM6SF2 on non-alcoholic fatty liver disease (NAFLD) in a Chinese population. Front Genet. 2016;7:140.

15. Chen LZ, Ding HY, Liu SS, Liu Q, Jiang XJ, Xin YN, Xuan SY. Combining I148M and E167K variants to improve risk prediction for nonalcoholic fatty liver disease in Qingdao Han population, China. Lipids Health Dis. 2019;18:45.

16. Posadas-Sanchez R, Lopez-Uribe AR, Posadas-Romero C, Perez-Hernandez N, Rodriguez-Perez JM, Ocampo-Arcos WA, Fragoso JM, Cardoso-Saldana G, Vargas-Alarcon G. Association of the I148M/PNPLA3 (rs738409) polymorphism with premature coronary artery disease, fatty liver, and insulin resistance in type 2 diabetic patients and healthy controls. The GEA study. Immunobiology. 2017;222:960-6.

17. Ruschenbaum S, Schwarzkopf K, Friedrich-Rust M, Seeger F, Schoelzel F, Martinez Y, Zeuzem S, Bojunga J, Lange CM. Patatin-like phospholipase domain containing 3 variants differentially impact metabolic traits in individuals at high risk for cardiovascular events. Hepatol Commun. 2018;2: 798-806.

18. Pirola CJ, Sookoian S. The dual and opposite role of the TM6SF2-rs58542926 variant in protecting against cardiovascular disease and conferring risk for nonalcoholic fatty liver: a meta-analysis. Hepatology. 2015;62:1742-56.

19. Rickham PP. Human experimentation. Code of Ethics of the World Medical Association Declaration of Helsinki. Br Med J. 1964;2:177.
20. Auton A, Brooks LD, Durbin RM, Garrison EP, Kang HM, Korbel JO, Marchin JL, McCarthy S, McVean GA, Abecasis GR. A global reference for human genetic variation. Nature. 2015;526:68-74.

21. Brouwers M, Simons N, Stehouwer CDA, Koek GH, Schaper NC, Isaacs A. Relationship between nonalcoholic fatty liver disease susceptibility genes and coronary artery disease. Hepatol Commun. 2019;3:587-96.

22. Pirazzi C, Adiels M, Burza MA, Mancina RM, Levin M, Stahlman M, Taskinen MR, Orho-Melander M, Perman J, Pujia A, et al. Patatin-like phospholipase domain-containing 3 (PNPLA3) I148M (rs738409) affects hepatic VLDL secretion in humans and in vitro. J Hepatol. 2012;57:1276-82.

23. Musso G, Cassader M, Gambino R. PNPLA3 rs738409 and TM6SF2 rs58542926 gene variants affect renal disease and function in nonalcoholic fatty liver disease. Hepatology. 2015;62:658-9.

24. Oniki K, Saruwatari J, Izuka T, Kajiwara A, Morita K, Sakata M, Otake K, Ogata Y, Nakagawa K. Influence of the PNPLA3 rs738409 polymorphism on nonalcoholic fatty liver disease and renal function among Normal weight subjects. PLoS One. 2015;10:e0132640.

25. Tonelli M, Muntner P, Lloyd A, Manns BJ, Klarenbach S, Pannu N, James MT, Hemmelgarn BR. Risk of coronary events in people with chronic kidney disease compared with those with diabetes: a population-level cohort study. Lancet. 2012;380:807-14.

\section{Publisher's Note}

Springer Nature remains neutral with regard to jurisdictional claims in published maps and institutional affiliations.
Ready to submit your research? Choose BMC and benefit from:

- fast, convenient online submission

- thorough peer review by experienced researchers in your field

- rapid publication on acceptance

- support for research data, including large and complex data types

- gold Open Access which fosters wider collaboration and increased citations

- maximum visibility for your research: over $100 \mathrm{M}$ website views per year

At $\mathrm{BMC}$, research is always in progress.

Learn more biomedcentral.com/submissions 